\title{
Management of Pancreatico-duodenal arterio-venous malformation
}

\author{
Clement Marcelin', Auh Whan Park², Patrick Gilbert ${ }^{1}$, Louis Bouchard', Eric Therasse ${ }^{1}$, Pierre Perreault', \\ Marie France Giroux ${ }^{1}$ and Gilles Soulez ${ }^{1 *}$ (D)
}

\begin{abstract}
Purpose: To describe the interventional management and clinical outcome of pancreatico-duodenal arterio-venous malformations (PDAVMs).

Material and Methods: Seven patients presenting a PDAVM (6 women, 1 male; mean age: 61 ) were retrospectively reviewed. Technical, clinical success and complications of embolization and surgical management of symptomatic PDAVMs were assessed. Technical success was defined as a complete occlusion of the PDAVM and clinical success as no clinical symptom or recurrence during follow-up. Patients with asymptomatic PDAVMs were followed clinically, by Doppler ultrasound and CT-angiography.
\end{abstract}

Results: Mean follow-up time was 69 months (15-180). Five symptomatic patients presented with upper gastrointestinal bleeding $(n=3)$, ascites $(n=1)$, and abdominal pain $(n=1)$. Two patients were asymptomatic. The PDAVMs were classified as follow: Yakes I (1), IIla (2), IIlb (3) and IV (1). Five symptomatic patients were treated with 9 embolization sessions with arterial approach (onyx ${ }^{\oplus}$, glue, coils) in 7 and venous approach in 2 (plugs, coils, covered stents, STS foam and ony $x^{\oplus}$ ). Technical success of embolization was $60 \%$ (3/5). Devascularization was incomplete for 2 Yakes IIIB patients. Clinical success of embolization was estimated at 80\% (4/5) as one patient required additional surgery (Whipple) because of persistent bleeding. One splenic vein thrombosis was treated successfully by mechanical thrombectomy and heparin. No recurrence occurred during follow-up. No progression was documented in asymptomatic patients.

Conclusion: Embolization of symptomatic PDAVMs is effective and surgery should be performed in second intention. Complete devascularization is more difficult to obtain in Yakes III PDAVM.

Keywords: Embolization, AVM, Pancreas, Percutaneous

\section{Introduction}

Pancreatico-duodenal arterio-venous malformations (PDAVMs) are rare but a proper diagnosis is important to differentiate them from hypervascular tumors (Shearer et al., 2011) . PDAVMs are mostly congenital, and can be associated with Hereditary Hemorrhagic Telangiectasia, commonly known as Osler-Weber-Rendu Syndrome (Lacout et al., 2010).

\footnotetext{
* Correspondence: gilles.soulez.med@ssss.gouv.qc.ca

${ }^{1}$ CHUM Université de Montréal, Montreal, Québec, Canada

Full list of author information is available at the end of the article
}

PDAVMs are often asymptomatic, but complications can arise, such as gastrointestinal bleeding, abdominal pain, portal hypertension and pancreatitis (Koito et al., 2001). CT is useful to make the diagnosis and identify feeding arteries and draining veins (Ogawa et al., 2009), however arteriography remains the gold standard.

There is no consensus on the management of asymptomatic PDAVMs (Chou et al., 2013) . However, symptomatic PDAVMs can be treated by embolization and/or surgery (Chou et al., 2013; Song et al., 2012).

In previous case reports PDAVM embolization was technically successful in $84 \%$ of cases and led to clinical improvement in $100 \%$ of patients (Cassinotto \& 
Lapuyade, 2015; Yamamoto et al., 2008; Frenk et al., 2016; Grasso et al., 2012) . Surgery is effective (Song et al., 2012) but carries a high risk of intraoperative bleeding, and severe complications (Wang et al., 2018).

Yakes and Cho classification helps for determining proper treatment of patients affected by AVMs, by describing the angioarchitecture of the AVM (Soulez et al., 2019; Yakes \& Baumgartner, 2014). It will dictate the approach to eradicate the nidus of the AVM (intra-arterial, venous retrograde or direct puncture). Careful selection of the approach is important to reach the nidus while minimizing the risk of reflux of embolizing agents into normal arteries and veins.

The purpose of study is to present a series of PDAV Ms and describe the safety and the efficacy of embolization according to the Yakes classification.

\section{Material and methods}

\section{Study design}

The Institutional Review Board approved this study and waived signed informed consent for this retrospective study. Data were collected through review of medical and imaging reports.

All patients with a diagnosis of PDAVM between 2000 and 2019 were included. A search with the following index terms was performed in the radiological information system: arteriovenous malformation and pancreas or duodenum, embolization, CT, Doppler ultrasound, CT, CT angiography.

\section{Transarterial embolization}

For all patients having embolization, transarterial procedures were performed under local anesthesia and conscious sedation. After percutaneous introduction of a 5-
Fr sheath in the right or left femoral artery, the celiac trunk and the superior mesenteric artery (SMA) were selectively catheterized using a 5F Chuang catheter and a hydrophilic guidewire 0.035 (Terumo ${ }^{\circ}$, Tokyo, Japan). Arteriography was performed to evaluate different feeders, localize the nidus, and visualize draining vein(s).

The nidus was accessed first by a trans-arterial approach using a microcatheter, either a Progreat Terumo $2.4 \mathrm{Fr}$, Tokyo, Japan) or a Renegade (Boston Scientific ${ }^{\circ}$, USA), as close as possible to the nidus of the vascular malformation. The arterial feeders were embolized with a liquid agent, chosen based on the operator experience. Ethylene Vinyl Alcohol Copolymer (Onyx 18 ${ }^{\circ}$, ev3 Irvine, CA, USA) (Fig. 1) or glue (Histoacryl; Braun Germany or Glubran; GEM, Italy) with lipiodol ${ }^{\circ}$ (Guerbet, France) dilution between 1-2 and 1-3, or 300-500 $\mu$ m PVA particles (when onyx $^{\circ}$ was not available). Coils (Concerto ${ }^{\circ}$, Medtronic, Target 360 Stryker, Fremont, CA, and Azur ${ }^{\circ}$ CX 18, Terumo) were used to protect distal arteries not feeding the AVM, to complete proximal embolization following distal liquid embolization or to decrease arterial flow in combination with a transvenous approach.

\section{Transvenous embolization}

Transvenous procedures were performed in 2 patients under general anesthesia because of failure of previous trans-arterial embolization.

In one case, the AVM located in the head of the pancreas was draining in the gastroduodenal vein. A percutaneous portal access was performed under ultrasound with a micropuncture set. A coaxial technique was used with deployment of an Amplatzer ${ }^{\circ}$ plug (Amplatzer ${ }^{\bullet}$, St Jude medical, Plymouth, Minnesotta) in the proximal portion of the gastroduodenal vein. Using a microcatheter (Progreat ${ }^{\circ} 2.7$,
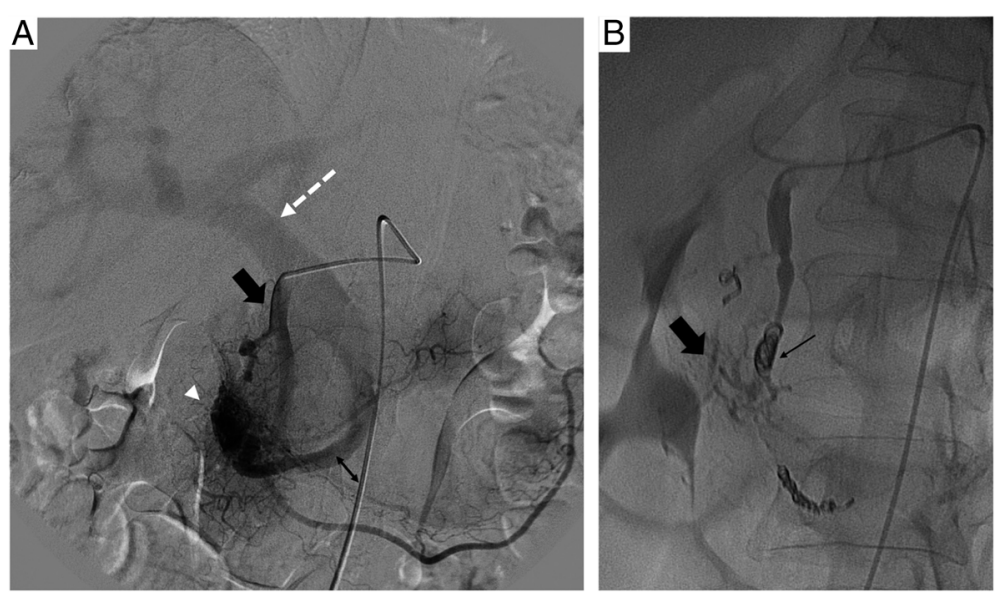

Fig. 1 A 79 yo women with upper Gl bleeding. A- Arteriography showed a type Illa pancreatic AVM, vascularized by the gastro-duodenal artery (black arrow), connected to the gastro-duodenal vein (double arrow) with a nidus (arrowhead), draining into the portal vein (dotted arrow). BAfter embolization of the gastroduodenal artery with coils (black arrow) and the nidus with onyx (large black arrow), opacification of the celiac trunk showed no residual vascularization of the AVM. 
Terumo, Tokyo, Japan) advanced distally to the plug, the connection between the gastroduodenal vein and the superior mesenteric vein was coiled to prevent reflux in the portal system. Then, the vein was embolized with foam made of two ml of Sodium Tetradecyl Sulfate (STS) 3\% (60mg) mixed with $1.5 \mathrm{ml}$ lipiodol and $4 \mathrm{ml}$ room air injected distally to the Amplatzer ${ }^{\circ}$ plug using the pressure cooker technique to reflux into the nidus (Chapot et al., 2014) (Fig. 2). In the second case, the AVM involving the tail of the pancreas was draining into the splenic vein, which was aneurysmal (Fig. 3). Since this patient has a chronic portal thrombosis, a transplenic access was performed. Two covered stent grafts were deployed (Viabahn, Gore, Newark, Deleware) of $6 \mathrm{~mm} \mathrm{X} 7.5 \mathrm{~cm}$ in the splenic vein and of 8 $\mathrm{mm} \mathrm{X} 2 \mathrm{~cm}$ at the spleno-mesenteric vein junction) to exclude the venous aneurysm while maintaining its patency. The aneurysm sac was then embolized using Onyx ${ }^{\circ}$ and coils after direct puncture of the aneurysm.

\section{Image analysis}

The PDAVMs were classified according to the Yakes classification (Yakes \& Baumgartner, 2014) (Fig. 4).

Technical success was defined as a complete occlusion of the PDAVM on the CT imaging on the follow-up. Clinical success was defined as resolution of symptoms after the embolization during the follow-up.

\section{Follow-up}

Clinical and imaging follow up was performed for all patients at the interventional radiology clinic. All CTs had at least an arterial and portal phase. For patients with an active bleeding a delayed venous phase was also performed. On CT, the criteria for diagnosing the AVM were the presence of enlarged arterial feeders and draining vein and the early filling of the portal system on the arterial phase.

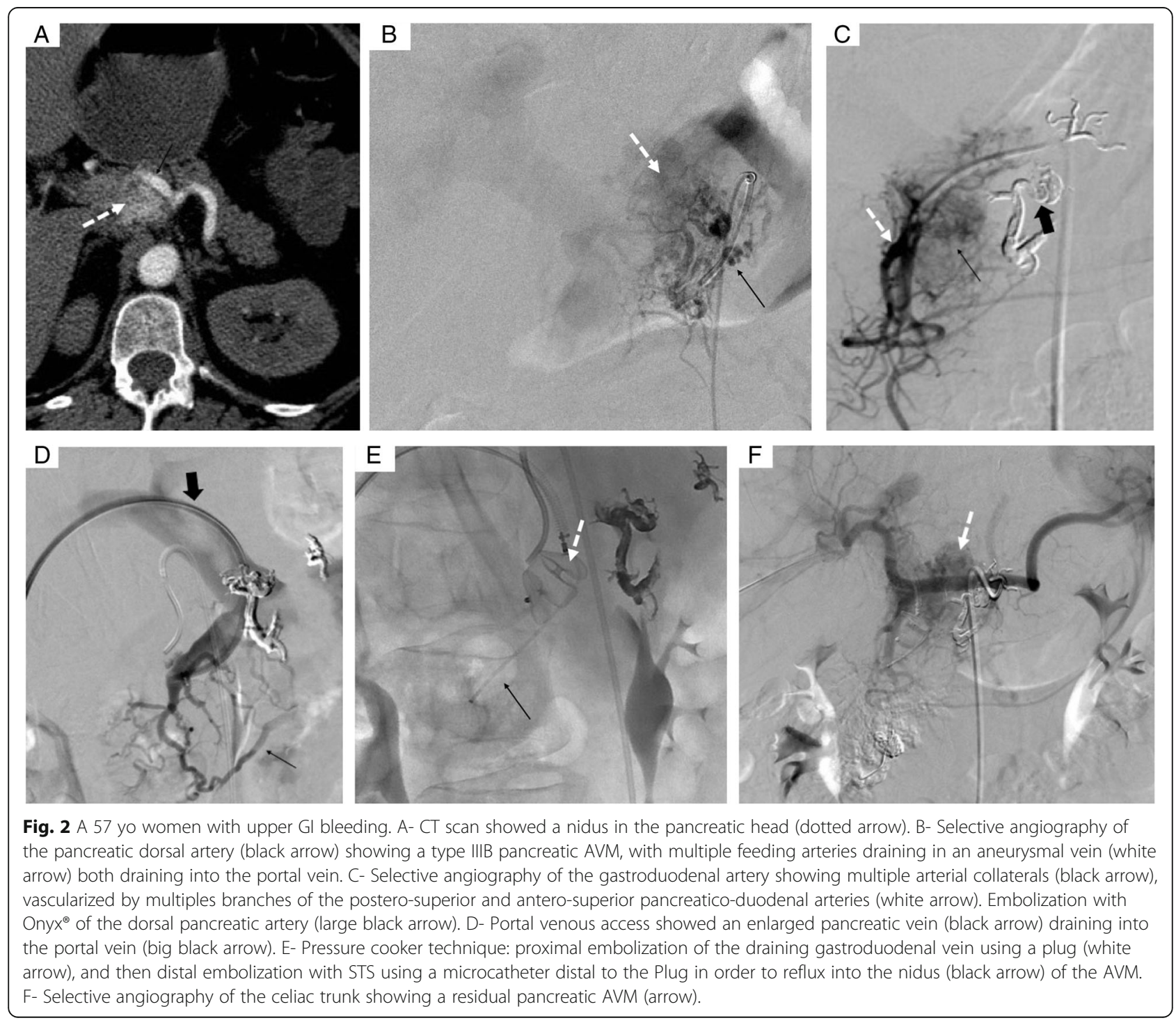



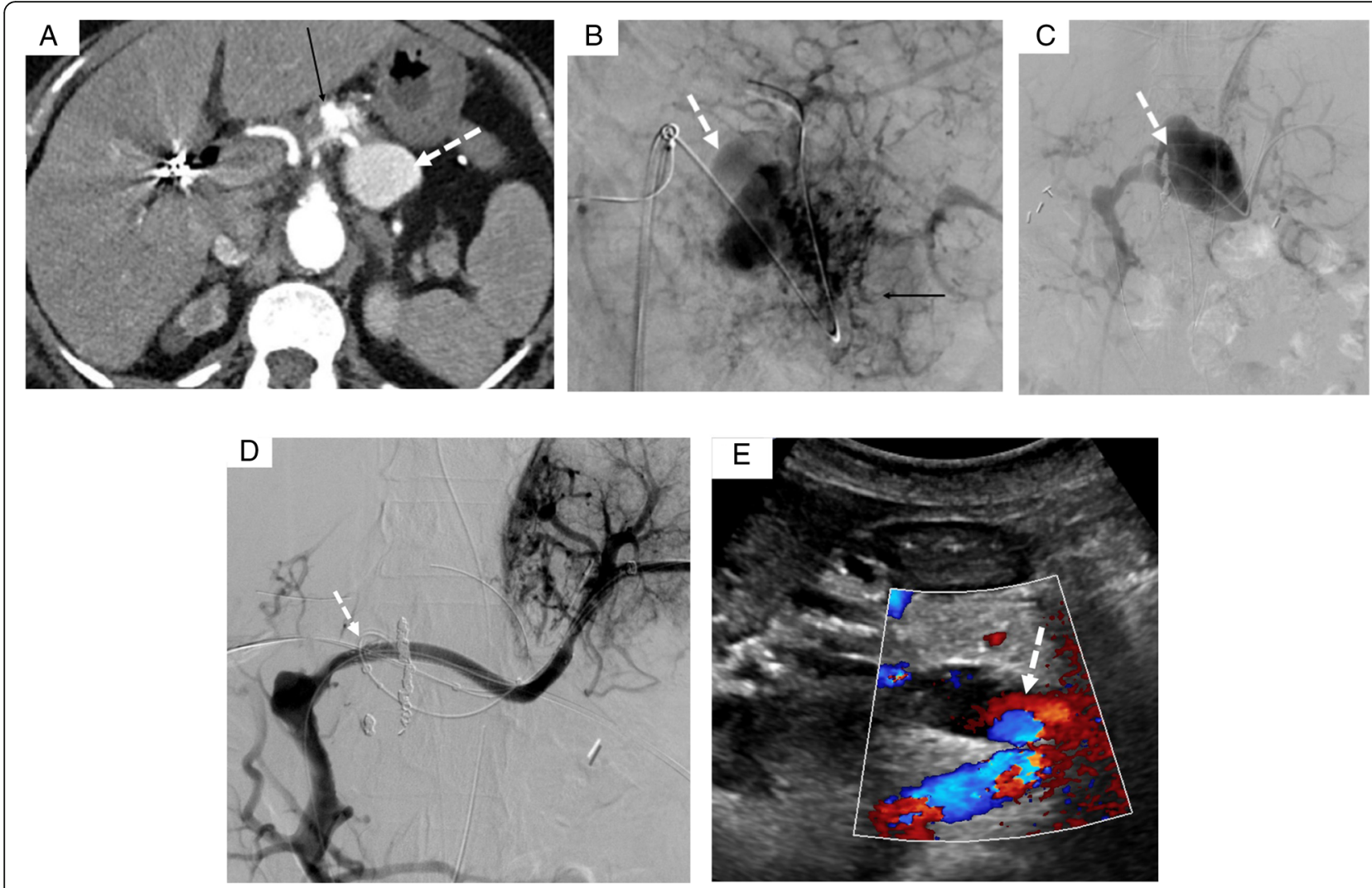

Fig. 3 A 66 yo women with a cryptogenic cirrhosis who underwent previous abdominal surgery for colorectal carcinoma and chronic portal vein thrombosis presented recurrent ascites and chronic pancreatitis. A CT scans showing a Yakes type Illa pancreatic AVM (arrow), with an aneurysmal splenic vein (dashed arrow). B Selective angiography of the splenic artery showed a pancreatic AVM, vascularized by the dorsal pancreatic artery (arrow), splenic artery, left gastric artery, and connected to an aneurysmal splenic vein (dashed arrow). C After puncture of splenic vein, venography showing the aneurysmal splenic vein draining into the gastroduodenal and mesenteric veins because of the preexisting portal thrombosis (dashed arrow). D Insertion of a covered stent in the splenic vein (dashed arrow) by a transplenic access and embolization using Ony $x^{\oplus}$ and coils after direct puncture of the aneurysm. The patient had subsequent splenic venous thrombosis which was successfully treated by mechanical thrombectomy and heparin infusion. E Doppler ultrasound at 1 year showed permeability of the splenic and portal veins (dashed arrow), with no residual AVM.

A routine post-embolization contrast-enhanced CT scan with non-contrast, arterial and portal phases, and doppler ultrasound were performed 1 to 6 months after the embolization. Additional intervention was performed in case of PDAVM recurrence. Asymptomatic patients were followed for a mean period of 2 years and had a control CT scan and doppler ultrasound at last followup. The utility of Doppler ultrasound is to document the magnitude of AV fistulae (aliasing in the shunting area and arterialization of the portal flow).

\section{Results}

\section{Patients}

Patient characteristics are summarized in Table 1. From 2000 to 2019, seven consecutive patients presenting a PDAVM (6 women, 1 man; mean age: 61.1, range 43-79) were analyzed. No patients were excluded during the study period. Two asymptomatic patients had no intervention and close imaging and clinical follow-up. Five symptomatic patients (2 massive upper gastrointestinal bleeding in emergency and 1 with repeated upper gastrointestinal bleeding for 3 patients, ascites for one, and persistent and resistant abdominal pain for one were treated with first intention embolization (1 male and 4 female). Patients presenting with massive upper gastrointestinal bleeding were initially imaged with CT (2 emergency procedure), and the others were imaged with CT and Doppler ultrasound. Mean follow-up time was 69 months (15-180).

\section{Imaging findings}

On CT and catheter angiography, the feeding arteries included the gastroduodenal artery $(n=1)$, the anterior superior pancreatico-duodenal artery $(n=1)$, the posterior superior pancreatico-duodenal arteries $(n=4)$, the anterior inferior pancreatico-duodenal artery $(n=1)$, the posterior inferior pancreatico-duodenal arteries $(n=5)$, the great pancreatic arteries (pancreatica magna) $(n=2)$, the 


\begin{tabular}{ll}
\hline Type & Criteria \\
\hline Type I & Direct AV fistula \\
Type II & $\begin{array}{c}\text { Typical AVM nidus } \\
\text { Multiple inflow arteries leading to nidus and vein outflow }\end{array}$
\end{tabular}

Type IIla Multiple in-flow arterioles shunting into an aneurysmal vein that has a single vein out-flow. Fistulae are in the vein wall

Type IIlb Multiple in-flow arterioles shunting into an aneurysmal vein with multiple out-flow veins. The fistulae (nidus) are in the vein wall

Typical Appearance
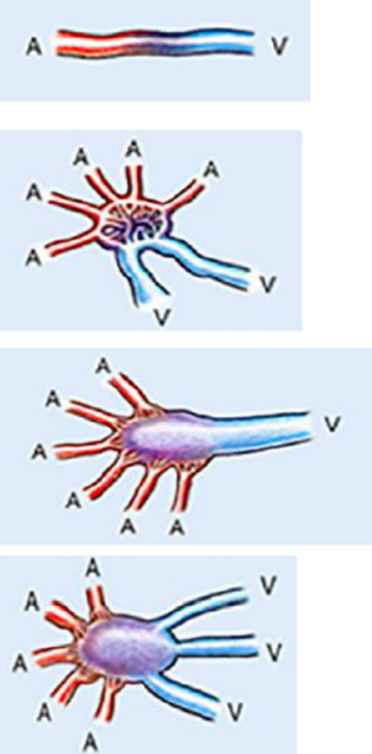

Type IV

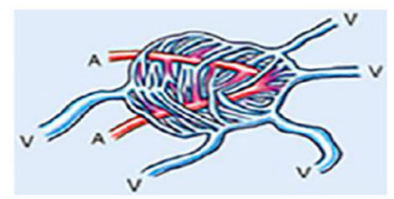
drainage then competes with the arterialized vein out-flow for drainage causing venous HTN in tissue.

Fig. 4 Yakes AVM classification (Soulez et al., 2019)

dorsal pancreatic artery, the splenic artery $(n=1)$, the hepatic artery $(n=1)$, the left gastric artery $(n=1)$.

Draining veins included the gastroduodenal vein $(n=$ $5)$, the splenic vein $(n=2)$, and the right gastric vein $(n=1)$.

\section{Clinical outcome}

Technical success of embolization was estimated at $60 \%(3 / 5)$. Devascularization was incomplete for 2 patients (25\% for one patient, and $75 \%$ for one patient), both in Yakes IIIB PDAVMs. Clinical success of embolization was estimated at $80 \%(4 / 5)$ (Yakes 1, 2 Yakes 3a, Yakes 3b) as one patient (Yakes IIIB) required additional surgery (Whipple) because of persistent bleeding. This patient had a partial embolization (successive arterial and venous approaches) estimated at $25 \%$ devascularization.

One splenic vein thrombosis (Yakes IIIA), occurred after a stenting of aneurysmal splenic vein draining the AVM. It was successfully treated by mechanical thrombectomy followed by an overnight local infusion of heparin. The splenic vein thrombosis was treated to prevent an additional segmental portal hypertension to a chronic portal thrombosis with inherent risk of gastric or gastroesophageal bleeding.

No recurrence occurred during clinical and CT imaging follow-ups. One patient (Yakes IIIB) had 25\% residual AVM without symptom recurrence during the follow-up.

The patient with splenic vein thrombosis and preexisting portal thrombosis was anticoagulated for 12 months with good patency of the covered stent-grafts, without recurrence of AVM. There was no other complication and no evidence of deterioration of liver, pancreatic and renal function on blood tests. Patient hospitalization ranged between 24 hours and 7 days.

Asymptomatic patients presented with a Yakes IIIb and IV, and had no evidence of AVM progression and did not require additional treatment.

\section{Discussion}

In this study complete regression of symptoms of the AVM was reported at $80 \%$ while technical success of embolization was $60 \%$. One of the 2 Yakes IIIB patients 
Table 1 Patients characteristics

\begin{tabular}{|c|c|}
\hline Patient characteristics & Mean (range) or $\mathrm{N}(\%)$ \\
\hline Average age in years (range) & 61.1 (range 43-79) \\
\hline \multicolumn{2}{|l|}{ Gender } \\
\hline -Male & 1 \\
\hline -Female & 6 \\
\hline History of abdominal surgery, portal vein thrombosis, cirrhosis or pancreatitis & 1 \\
\hline \multicolumn{2}{|l|}{ Symptoms } \\
\hline -hemorrhage & 5 \\
\hline -ascites & 1 \\
\hline -abdominal pain & 1 \\
\hline -none & 2 \\
\hline \multicolumn{2}{|l|}{ Classification Yakes } \\
\hline-1 & 1 \\
\hline$-\| l a$ & - \\
\hline$-\| \mathrm{b}$ & - \\
\hline-||$l \mid a$ & 2 \\
\hline$-|l| b$ & 3 \\
\hline$-\mathrm{IV}$ & 1 \\
\hline \multicolumn{2}{|l|}{ Localization } \\
\hline -head & 3 \\
\hline -isthmus + head & 2 \\
\hline -head+isthmus+body & 1 \\
\hline -body + isthmus & 1 \\
\hline Mean size of the nidus (mm) & 24.5 (range 20-30) \\
\hline \multicolumn{2}{|l|}{ Treatment } \\
\hline -none & 2 \\
\hline -embolization & 5 \\
\hline -surgery & 1 \\
\hline
\end{tabular}

with incomplete embolization (25\%) required additional surgery whereas the other with a $75 \%$ devascularization had a good evolution with conservative treatment.

Classification of AVMs is useful for planning optimal therapeutic approach, and predicting therapeutic outcomes (Yakes et al., 1996; Cho et al., 2006). Cho et al. (2006) demonstrated better outcome in type II Cho AVMs involving extremities or the thoraco-abdominal region.

Yakes classification was preferred to Cho classification in this study because it was more representative of the angioarchitecture observed on imaging. The Yakes classification is more comprehensive especially the distinction between type IIIa and III b which applied for $42 \%$ (3/7) of our patients.

Six of seven patients had no history of previous abdominal surgery, splenic or portal vein thrombosis or pancreatitis, these patients probably had in-born AVMs discovered at the adult age. The patient showed in Fig. 3 who had a previous abdominal surgery, portal thrombosis and chronic pancreatitis could have an acquired AVM.

Ideally, the embolization endpoint is to obtain a complete nidus occlusion of the PDAVM. Transarterial approach was used in first intention to embolize the PDAVM. Transvenous approach was selected in second intention following failure of transarterial approaches or when multiple arterial feeders were present with a dominant draining vein.

Transvenous approach was useful, however embolization should be performed carefully to minimize the risk of splenic or portal thrombosis. We observed one case of the latter, which was successfully managed by mechanical thrombectomy and local heparin infusion.

Different embolic agents have been used in previous reports: particles and ethanol were described with good technical success (Frenk et al., 2016), embolization with Ony $x^{\oplus}$ showed complete regression of PDAVMs 
(Cassinotto \& Lapuyade, 2015; Grasso et al., 2012), and Glue utilized as well (Tatsuta et al., 2014). Liquid embolic agents are the most useful. Onyx ${ }^{\circ}$ provides the best control for liquid agent and should be preferred to minimize the risk of non-target embolization (Guimaraes \& Wooster, 2011). The speed of polymerization of glue is more difficult to predict and the slow injection rate of Onyx ${ }^{\circ}$ give more chance to the operator to prevent non target embolization (Li \& Barthes-Biesel, 2017). Glue had a higher risk of bowel infarction and glue reflux into other vessels may result in non-target embolization. On the venous side, the combination of mechanical occlusion with coils or plugs with a liquid agent is recommended (Soulez et al., 2019; Lee et al., 2019). Insertion of a covered stent-graft combined with embolization of a venous aneurysm is an elegant approach to maintain the patency of splanchnic veins in case of an aneurysmal draining vein ( Yakes IIB, IIIA) (Beyer et al., 2015). Ethanol embolization has the potential to cure the AVM, however it carries a higher-risk of non-target embolization and potential gastroduodenal ulcer or pancreatitis.

Reported complications included gastric ulcer, pancreatitis, portal vein thrombosis, bowel ischemia (Lee, 2020). The occurrence of duodenal ulceration following the arterial approach being the most frequent (Cassinotto \& Lapuyade, 2015; Grasso et al., 2012). Selective TAE with NBCA in the pancreas caused localized ischemic necrosis without clinically significant pancreatitis in a swine model (Okada et al., 2012).

The transvenous approach for PDAVM was not reported yet. This approach carries a risk of portal, mesenteric or splenic vein thrombosis. It is important to control reflux of liquid embolic agent into the portal system by occluding potential re-entry in the portal system. This could be achieved by occluding draining veins connected to the portal system with plugs or coils. Intranidal sclerotherapy by direct puncture was not performed because the AVM were deeply located and difficult to image under ultrasound or non-contrast or delayed contrast enhanced CT. Moreover, this requires to puncture through digestive structures in most cases.

In an emergent setting, microparticles were used because the operator was not used to liquid embolics. Using 300-500um particles, there was no significant complication as previously reported by Aina et al for gastroduodenal bleeding (Aina et al., 2001).

Additional surgery could be proposed in case of persistent of symptomatic AVM after embolization.

Radiotherapy has been reported (Kishi et al., 2011), in asymptomatic patients, showing shrinkage of the AVM. TIPS was described in case of failure of PDAVM embolization, with good efficacy (Hayashi et al., 1998).
Total or partial pancreatectomy is the only complete cure treatment, however there is a risk of massive intraoperative bleeding, hypoglycemia, and pancreatic juice leakage (Song et al., 2012).

The limitations of the study were the small number of patients and the retrospective design using different embolic agents. Particles were used only in emergency when the interventionalist in charge was not comfortable with the use of liquid embolics. Imaging follow-up were made by $\mathrm{CT}$, thus, small residual AVMs with minimal AV shunting may have been overlooked. However, all patients did not have bleeding recurrence or evidence of portal hypertension during the follow-up. PDAVMs are rare, making a prospective study impossible in a reasonable time frame.

In conclusion, embolization of PDAVMs is a safe approach and can be effective. In case of persistent symptomatic PDAVM after embolization, surgery is a valid option. Yakes Grade IIIb PDAVM are more challenging and partial devascularization can be observed.

\section{Authors' contributions}

CM was in charge of data collection and drafting the manuscript, AWP, PG, $E T, L B, P P$ and MFG were involved in interventional procedures, manuscript revision and editing. GS was involved in interventional procedures, manuscript revision and takes primary responsibility of the study results. The authors read and approved the final manuscript. The authors declare they have no competing interests.

\section{Author details}

${ }^{1} \mathrm{CHUM}$ Université de Montréal, Montreal, Québec, Canada. ${ }^{2}$ Department of Radiology, UVA Health, Charlottsville, VA, USA.

Received: 26 August 2021 Accepted: 16 November 2021 Published online: 03 January 2022

\section{References}

Aina R, Oliva VL, Therasse É, Perreault P, Bui BT, Dufresne M-P et al (2001) Arterial Embolotherapy for Upper Gastrointestinal Hemorrhage: Outcome Assessment. J Vasc Interv Radiol 12(2):195-200

Beyer LP, Wohlgemuth WA, Uller W, Pregler B, Goessmann H, Niessen C et al (2015) Percutaneous treatment of symptomatic superior mesenteric vein stenosis using self-expanding nitinol stents. Eur J Radiol 84(10):1964-1969

Cassinotto C, Lapuyade B (2015) Pancreatic Arteriovenous Malformation Embolization with Onyx. J Vasc Interv Radiol 26(3):442-444

Chapot R, Stracke P, Velasco A, Nordmeyer H, Heddier M, Stauder M et al (2014) The Pressure Cooker Technique for the treatment of brain AVMs. J Neuroradiol 41(1):87-91

Cho SK, Do YS, Shin SW, Kim D-I, Kim YW, Park KB et al (2006) Arteriovenous Malformations of the Body and Extremities: Analysis of Therapeutic Outcomes and Approaches According to a Modified Angiographic Classification. J Endovasc Ther 13(4):527-538

Chou S-C, Shyr Y-M, Wang S-E (2013) Pancreatic Arteriovenous Malformation. J Gastrointest Surg 17(7):1240-1246

Frenk NE, Chao TE, Cui J, Fagenholz PJ, Irani Z (2016) Staged Particle and Ethanol Embolotherapy of a Symptomatic Pancreatic Arteriovenous Malformation. J Vasc Interv Radiol 27(11):1734-1735

Grasso RF, Cazzato RL, Luppi G, Faiella E, Del Vescovo R, Giurazza F et al (2012 Aug) Pancreatic Arteriovenous Malformation Involving the Duodenum Embolized with Ethylene-Vinyl Alcohol Copolymer (Onyx). Cardiovasc Intervent Radiol 35(4):958-962

Guimaraes M, Wooster M (2011) Onyx (Ethylene-vinyl Alcohol Copolymer) in Peripheral Applications. Semin Interv Radiol 28(3):350-356

Hayashi N, Sakai T, Kitagawa M, Inagaki R, Yamamoto T, Ishii Y et al (1998) Intractable gastrointestinal bleeding caused by pancreatic arteriovenous 
malformation: successful treatment with transjugular intrahepatic portosystemic shunt. Eur J Radiol 28(2):164-166

Kishi K, Shirai S, Sato M, Sonomura T (2011) Role of external beam radiotherapy for arteriovenous malformation of the pancreas. Jpn J Radiol 29(7):517-520

Koito K, Namieno T, Nagakawa T, Ichimura T, Hirokawa N, Mukaiya M et al (2001) Congenital Arteriovenous Malformation of the Pancreas: Its Diagnostic Features on Images. Pancreas 22(3):267-273

Lacout A, Pelage J-P, Lesur G, Chinet T, Beauchet A, Roume J et al (2010) Pancreatic Involvement in Hereditary Hemorrhagic Telangiectasia: Assessment with Multidetector Helical CT. Radiology 254(2):479-484

Lee SM (2020) Transcatheter Arterial Embolization for Gastrointestinal Bleeding related to Pancreatic Adenocarcinoma: Clinical Efficacy and Predictors of Clinical Outcome. Eur J Radiol 123:108787

Lee SY, Do YS, Kim CW, Park KB, Kim YH, Cho YJ (2019) Efficacy and Safety of Transvenous Embolization of Type II Renal Arteriovenous Malformations with Coils. J Vasc Interv Radiol 30(6):807-812

Li YJ, Barthes-Biesel D (2017) Polymerization kinetics of n-butyl cyanoacrylate glues used for vascular embolization. J Mech Behav Biomed Mater 69:307-317

Ogawa H, Itoh S, Mori Y, Suzuki K, Ota T, Naganawa S (2009) Arteriovenous malformation of the pancreas: assessment of clinical and multislice CT features. Abdom Imaging 34(6):743-752

Okada T, Yamaguchi M, Takahashi T, Izaki K, Uotani K, Sakamoto N et al (2012) Is Embolization of the Pancreas Safe? Pancreatic Histological Changes after Selective Transcatheter Arterial Embolization with N-Butyl Cyanoacrylate in a Swine Model. Cardiovasc Intervent Radiol 35(1):161-167

Shearer DD, Demos TC, Sichlau MJ. Pancreatic Arteriovenous Malformation: a case report and literature review. J Radiol Case Rep. 2011;5(8):8-13.

Song KB, Kim SC, Park JB, Kim YH, Jung YS, Kim M-H et al (2012) Surgical Outcomes of Pancreatic Arteriovenous Malformation in a Single Center and Review of Literature. Pancreas 41(3):388-396

Soulez G, Gilbert P, Giroux M-F, Racicot J-N, Dubois J (2019) Interventional Management of Arteriovenous Malformations. Tech Vasc Interv Radiol 22(4): 100633

Tatsuta T, Endo T, Watanabe K, Hasui K, Sawada N, Igarashi G et al (2014) A Successful Case of Transcatheter Arterial Embolization with N-butyl-2 cyanoacrylate for Pancreatic Arteriovenous Malformation. Intern Med 53(23): 2683-2687

Wang J, Ma R, Churilov L, Eleftheriou P, Nikfarjam M, Christophi C et al (2018 Mar) The cost of perioperative complications following pancreaticoduodenectomy: A systematic review. Pancreatol Off J Int Assoc Pancreatol IAP AI 18(2):208-220

Yakes W, Baumgartner I (2014 Jul) Interventional treatment of arterio-venous malformations. Gefässchirurgie 1(19):325-330

Yakes WF, Rossi P, Odink H (1996) How I do it. Arteriovenous malformation management. Cardiovasc Intervent Radiol 19(2):65-71

Yamamoto K, Tominaga K, Kanke K, lijima M, Abe A, Shimoda M et al (2008) Hepatobiliary and pancreatic: Arteriovenous malformation of the pancreas. J Gastroenterol Hepatol 23(3):498-498

\section{Publisher's Note}

Springer Nature remains neutral with regard to jurisdictional claims in published maps and institutional affiliations.

\section{Submit your manuscript to a SpringerOpen ${ }^{\circ}$ journal and benefit from:}

- Convenient online submission

- Rigorous peer review

- Open access: articles freely available online

- High visibility within the field

- Retaining the copyright to your article

Submit your next manuscript at $\boldsymbol{\nabla}$ springeropen.com 